\title{
Analyse de la période représentative de la houle en zone de surf
}

\author{
Nadia Sénéchal $^{(1)}$, Philippe Bonneton ${ }^{(2)}$, Hélène Dupuis ${ }^{(2)}$ \\ ${ }^{1}$ Doctorante, Département de Géologie et Océanographie, Université \\ de Bordeaux I, avenue des Facultés, 33405 Talence, cedex. + 33(0)5 \\ 568483 81.n.senechal@geocean.u-bordeaux.Fr \\ ${ }^{2}$ Chargé de recherche, UMR 5805-EPOC, Université de Bordeaux I, \\ avenue des Facultés, 33405 Talence, cedex. dupuis@geocean.u- \\ bordeaux.fr. p.bonneton@geocean.u-bordeaux.Fr
}

Résumé

La période de la houle est un paramètre clés dans la plupart des modèles long terme qui ont pour but la prévision de l'évolution du trait de côte. Cet article présente deux phénomènes (génération d'harmoniques et fusion de fronts d'ondes) qui modifient significativement la distribution de la période des vagues sur une courte distance en zone de surf, là où justement la houle joue un rôle majeur dans le transport sédimentaire et l'évolution du trait de côte. Cet article met également en avant la difficulté à caractériser correctement la période représentative de la houle à partir d'un spectre dans cette zone.

Abstract

The significant wave period is a key parameter in many long term morphodynamic models but also in coastal engineering. In this paper, two phenomena (secondary wave generation and merging shocks) leading to significant wave period distribution modifications will be shown. Emphasis will also be placed on the difficulty of determining the significant wave period from a sea surface elevation energy density spectrum in the surf zone.

\section{Introduction}

\subsection{L'enjeu actuel de la zone littorale}

La gestion de la zone littorale est devenue une priorité pour de nombreuses collectivités locales. En effet, cette zone a un double enjeu: écologique mais également socio-économique, et de ce fait elle est soumise à des pressions intenses. La densité moyenne des communes littorales est près de deux fois et demie supérieure à la moyenne nationale. L'artificialisation du littoral s'est effectuée, entre 1975 et 1990, à un rythme soutenu : sur une bande de $10 \mathrm{~km}$, le 
taux de croissance approche les $15 \%$ (données IFE). Or, sur les $3500 \mathrm{~km}$ de côte que compte environ la France, $70 \%$ des plages sont en cours d'érosion et seulement $10 \%$ en cours d'extension.

Dans ce contexte, la compréhension des processus de type petites échelles (physiques, biogéochimiques) et des transports particulaires et sédimentaires associés, à l'origine de phénomènes à plus grandes échelles (suivi de la morphologie du fond mais aussi évolution du trait de côte), constitue un préalable indispensable pour la gestion du littoral.

\subsection{Le rôle de la houle et son intégration dans les modèles long terme}

La houle joue un rôle particulièrement important dans le transport sédimentaire à l'approche de la côte et notamment dans la zone de déferlement puis dans la zone de surf. En effet, les conditions hydrodynamiques liées à la houle se trouvent fortement modifiées en faible profondeur sous l'action combinée de multiples processus (influence de la bathymétrie, réflexion, réfraction, déferlement, effets non-linéaires....), dont la plupart contrôlent la dissipation ou la concentration d'énergie de la houle incidente.

En ingénierie, mais également dans de nombreux modèles long terme de comportement, l'effet des vagues est paramétrisé soit par des formules analytiques, soit par des formules empiriques faisant généralement intervenir la hauteur et la période caractéristiques des vagues. Il existe notamment un certain nombre de formules d'ingénierie côtière, empiriques, qui permettent de calculer la dérive littorale annuelle, le long d'une côte sableuse. Or, les formules les plus couramment utilisées dans la littérature (formule du L.C.H.F., 1979 ; formule du C.E.R.C., 1984 ; formule de Bailard, 1984; formule de Kamphuis, 1993) supposent que la période caractéristique de la houle se conserve du large jusqu'à la côte.

Les résultats présentés dans ce travail s'inscrivent dans les objectifs de l'action de recherche «Dynamique Sédimentaire Côtière» dans le cadre du Programme National d'Environnements Côtiers. Ils mettent en évidence la difficulté à correctement estimer l'effet de la houle compte tenu que celle-ci peut se trouver fortement modifiée sous l'action de phénomènes non-linéaires. Ces résultats se basent sur des données acquises sur le site atelier du Truc Vert au cours de deux campagnes de mesures : en mai 1998 et en mars 2000.

\section{Les données de terrain}

\subsection{Le site atelier du Truc vert}

La plage du Truc Vert est située environ $10 \mathrm{~km}$ au Nord de la pointe du Cap Ferret. C'est une plage méso-macrotidale composée de sable moyen et soumise à une houle océanique, principalement de secteur $O$, NO. Pendant la période hivernale, les hauteurs significatives sont de l'ordre de $1.55 \mathrm{~m}$ pour une période significative de $12 \mathrm{~s}$ alors que pendant les conditions estivales, les hauteurs significatives sont de l'ordre de $0.9 \mathrm{~m}$ pour un période de $7 \mathrm{~s}$. 
Durant les périodes de conditions estivales, la plage du Truc Vert présente des systèmes barre-baïne en bas de zone intertidale qui sont ensuite partiellement ou totalement détruits pendant les conditions hivernales (Lafon et al., 2001).

\subsection{Acquisitions des données}

Les résultats présentés dans ce travail ont été obtenus à partir de mesures acquises au cours de deux campagnes de terrain (mai 1998 et mars 2000) dans le cadre du P.N.E.C.. Pour ces deux campagnes, les capteurs ont été déployés uniquement en zone intertidale (sauf pour mai 1998 où une bouée de type $\mathrm{S} 4$ a été mouillée par $10 \mathrm{~m}$ de profondeur) suivant une ligne perpendiculaire au trait de côte, de la laisse de basse mer jusqu'en haut de plage. Les capteurs étaient soit des capteurs de pression piézorésistifs (P1, P2, P3), soit des «Accoustic Doppler Velocimeter » (ADV Vector) soit des «Directionnal Wave Current Meter» de type S4 (S1, S2). Les acquisitions de données (à des fréquences de $2 \mathrm{~Hz}$ jusqu'à $32 \mathrm{~Hz}$ ) ont été synchronisées afin de permettre un suivi vague à vague le long de la chaîne d'acquisition. Ces deux expériences sont présentées en détail dans Sénéchal et al. $(2001 b, 2002)$.

\subsection{Les données de l'étude}
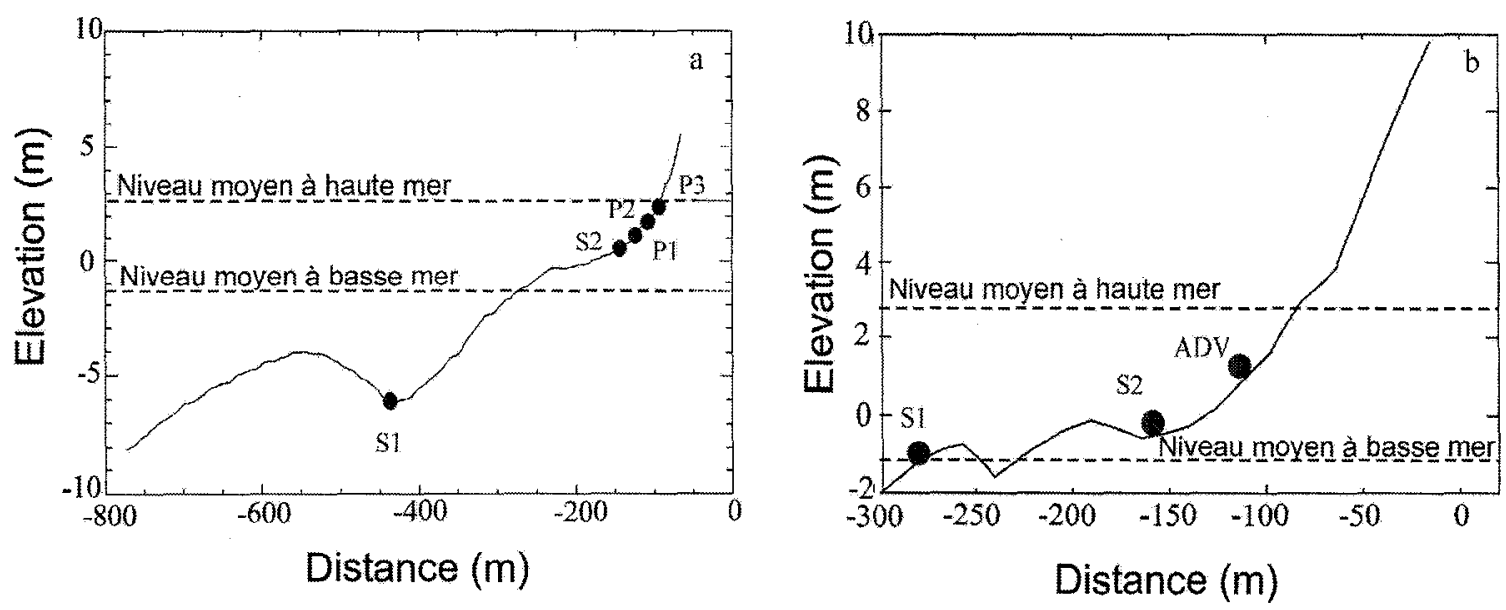

Figure 1. profil de plages instrumenté, (a) : mai 98 et (b) : mars 00. Figure 1. sensor line deployment, (a) : May 98 and (b) : March 00.

Deux jeux de données ont été sélectionnés, pour lesquels, d'une part le profil de plage au niveau de la ligne de capteurs (figure 1) et d'autre part le forçage hydrodynamique (figure 2) étaient différents : la journée du 28 mai 1998 avec une ligne de capteurs située en dehors du système barre-baïne et des conditions hydrodynamiques de type «mer du vent» irrégulière et peu énergétique ; et les journées des 20 et 21 mars 2000 avec un système de deux barre-baïne bien développé et une houle longue régulière et peu énergétique.

La figure 1 représente les profils de plage instrumentés pour (a) mai 98 et (b) mars 00 . 
La figure 2 illustre les deux forçages hydrodynamiques observés en (a) mai 1998 et (b) Mars 2000. En mai 1998 (figure 1a), on distingue une large bande énergétique qui s'étend entre $0.1 \mathrm{~Hz}(10 \mathrm{~s})$ et $0.2 \mathrm{~Hz}(5 \mathrm{~s})$. La hauteur significative pour cette journée était de l'ordre de $0.75 \mathrm{~m}$ par $9 \mathrm{~m}$ de fond. En Mars 2000 (figure 1b), le spectre de densité d'énergie est très étroit, centré autour de $0.08 \mathrm{~Hz}$ $(14 \mathrm{~s})$. La hauteur significative pour cette période était de l'ordre de $1 \mathrm{~m}$ dans $4 \mathrm{~m}$ d'eau.
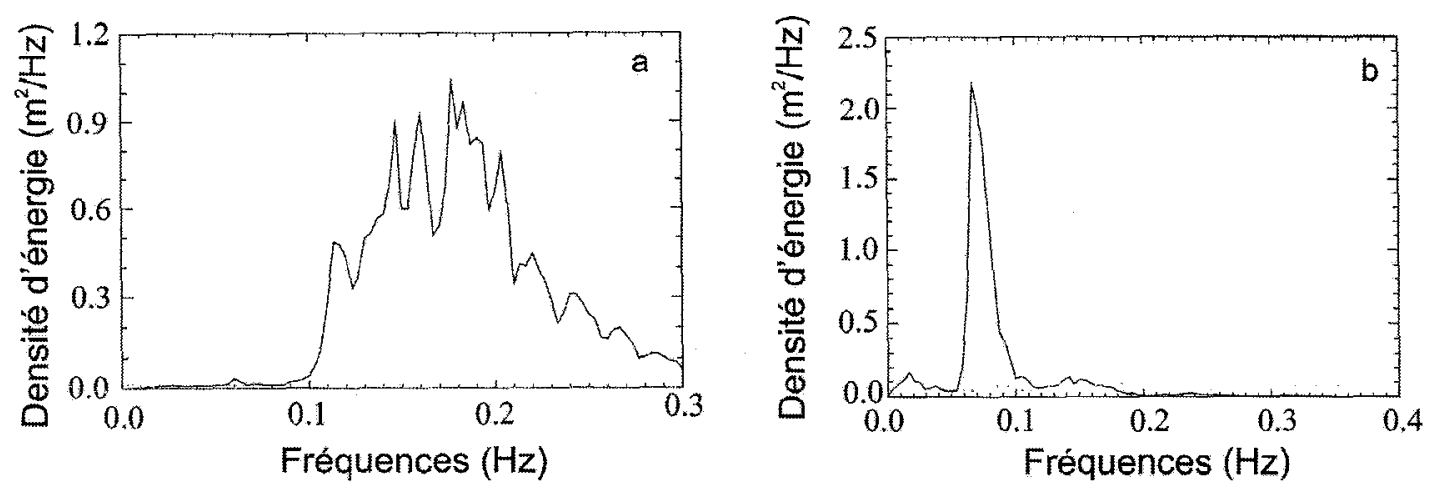

Figure 2. spectre de densité d'énergie mesuré à la bouée $\mathrm{S} 1$ : (a) mai 98 et (b) mars 00 .

Figure 2. energy density spectra as measured at sensor S1 : (a) May 98 et (b) March 00 .

Dans la prochaine partie, nous allons montrer que les caractéristiques de la houle ou de la mer du vent incidente se sont modifiées lorsque celles-ci se sont propagées dans la zone intertidale, en particulier nous allons nous intéresser à la modification de la répartition des périodes mais également à la modification du spectre au travers de deux mécanismes : la fusion de fronts d'ondes successifs et la génération d'harmoniques.

\section{Résultats}

\subsection{La fusion de front d'ondes}

Le phénomène de fusion de fronts d'ondes, qui est un phénomène non linéaire, est observable essentiellement en eau très peu profonde. On observe, en effet, un effet dispersif lié à la différence d'amplitudes entre les différentes ondes composants un groupe: les vagues se propagent d'autant plus vite que leur amplitude est grande. Sénéchal et al. (2001a) ont montré que ce phénomène était particulièrement significatif pour leur jeu de données de mai 1998. La première conséquence de ce phénomène est l'augmentation de la période moyenne des vagues lorsque celles-ci se propagent en zone de surf (figure 3 ).

La figure 3 illustre l'évolution de la répartition du rapport $T / T_{0}\left(a v e c T_{0}=\right.$ $T_{\text {rms }}$ au large) le long du profil instrumenté (figure 1a). On y observe clairement l'augmentation dans la zone de surf entre le capteur S2 et $\mathrm{P} 3$ de la période 
moyenne d'un facteur 1.2. Ce changement, significatif, a lieu sur une courte distance (environ $100 \mathrm{~m}$, soit quelques longueurs d'ondes).
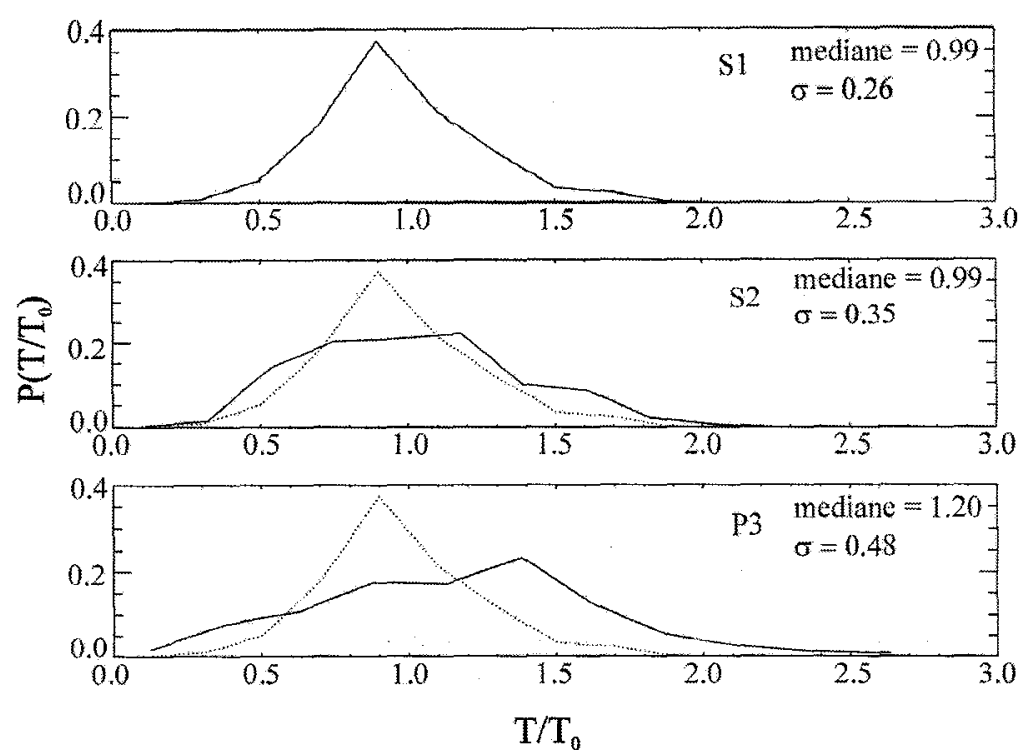

Figure 3. évolution de la répartition du rapport $\mathrm{T} / \mathrm{T}_{0}$ (avec $\mathrm{T}_{0}=\mathrm{T}_{\mathrm{rms}}$ au large) Figure 3. Observed probability density functions of ratio $T / T_{0}$ (where $T_{0}$ is $T_{r m s}$ in $9 m$ depth)

Parallèlement à l'augmentation de la période moyenne, on observe une augmentation continue du large (capteur S1) jusqu'à la plage (capteur P3) de la période significative de la houle d'un facteur 1.5 (Sénéchal et al., 2001a). L'évolution de la période significative de la houle est également perceptible au niveau spectral. Sénéchal et al. (2001b) ont montré que la fréquence centroïdale diminue dans la zone de surf d'environ $0.17 \mathrm{~Hz}$ au niveau du capteur S2 à 0.15 $\mathrm{Hz}$ au niveau de $\mathrm{P} 3$, suggérant une modification de la répartition énergétique dans le domaine fréquentiel.

Dans la partie suivante, nous allons évoquer un autre phénomène susceptible de modifier la période représentative de la vague : la génération puis la libération d'harmoniques.

\subsection{La génération d'harmoniques}

Lorsque la houle se propage en eau peu profonde, les effets non-linéaires s'intensifient, en particulier les interactions non-linéaires entre triplets de fréquences. D'un point de vue spectral, ces effets se traduisent par le transfert d'énergie de l'onde primaire $\left(\mathrm{F}_{\mathrm{p}}\right)$ vers les harmoniques $\left(\mathrm{nF}_{\mathrm{p}}\right)$ et/ou les subharmoniques. Lorsque le spectre incident est large, le transfert d'énergie se fait des ondes primaires $\left(F_{p, i}\right)$ vers les harmoniques associées $\left(\mathrm{nF}_{\mathrm{p}, \mathrm{i}}\right)$ mais également vers des fréquences résultant des interactions entre les différents modes primaires $\left(F_{p, i}+F_{p, j}\right)$. D'un point de vue temporel, ces effets se traduisent par la distorsion du profil des vagues : le front avant de la vague se raidit et la profil de vague devient fortement asymétrique. A cette étape, les harmoniques sont liées aux 
ondes primaires, c'est à dire qu'il existe une relation de phase entre les deux ondes.

Néanmoins, il a été mis en évidence, aussi bien dans des expériences de laboratoire (Beji et Battjes, 1993) que dans des expériences in-situ (Masselink, 1998 ; Sénéchal et al., 2002), que ces ondes liées pouvaient être libérées lorsque le champ de houle se propage au-dessus d'un obstacle immergé (rectangle, barre naturelle, récif..). La génération puis la libération de ces ondes engendrent un dédoublement (voir plus) des crêtes de vagues et une profonde modification du spectre incident.
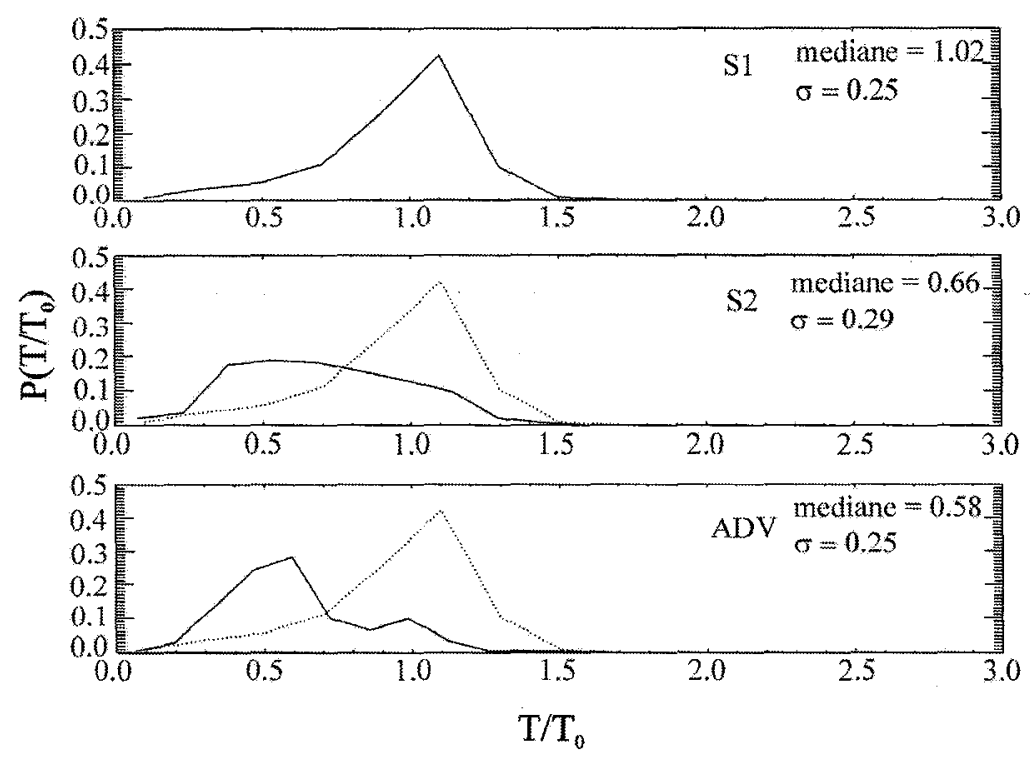

Figure 4. évolution de la répartition du rapport $\mathrm{T} / \mathrm{T}_{0}$ (avec $\mathrm{T}_{0}=\mathrm{T}_{\mathrm{rms}}$ au niveau du capteur S1)

Figure 4. Observed probability density functions of ratio $T / T_{0}$ (where $T_{0}$ is $T_{r m s}$ at sensor S1)

La figure 4, représentant l'évolution de la distribution de la période des vagues, met en évidence le dédoublement des crêtes des vagues lorsque celles-ci se propagent au-dessus du double système barre-baïne en bas de zone intertidale en mars 2000 (figure 1). On observe très nettement que la période des vagues est divisée par deux après les systèmes et ce sur quelques longueurs d'ondes (environ $150 \mathrm{~m}$ ). Sénéchal et al. (2002) ont montré que ce phénomène était lié à la libération des harmoniques et que ce phénomène avait lieu aussi bien dans le cas où l'on observe un point de déferlement sur la barre que dans le cas où l'on en observe pas. Cette figure illustre bien le risque d'erreur lorsque l'on considère la période au large pour appréhender des transports qui ont lieu essentiellement dans la zone de surf (LC.H.F., 1979 ; Kamphuis, 1993).

Dans le chapitre suivant, nous allons montrer la difficulté à correctement appréhender la période caractéristique de la houle à partir d'une représentation spectrale, lorsque celle-ci correspond à un signal fortement non-linéaire. Pour illustrer cela nous allons nous intéresser à un spectre caractéristique de la zone de surf pour l'expérience réalisée en mars 2000. 


\subsection{Période caractéristique à partir d'un spectre}

La figure 5 représente un spectre calculé au niveau du capteur ADV lorsque celui-ci était situé en zone de surf pendant l'expérimentation de mars 2000.

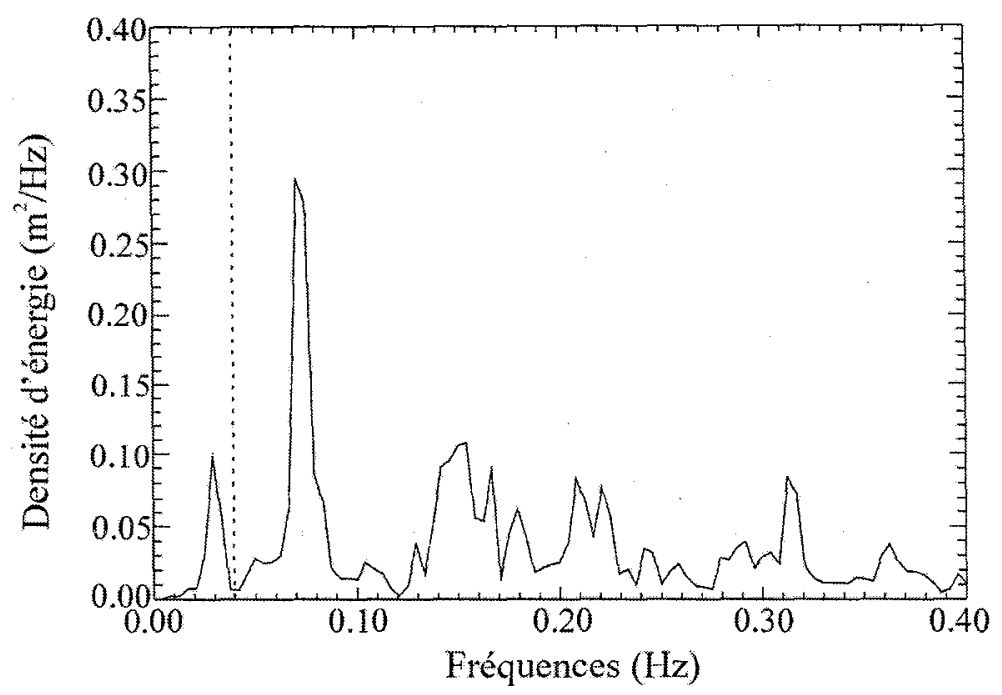

Figure 5. spectre d'énergie caractéristique en zone de surf au niveau de l'ADV pendant mars 2000.

Figure 5. typical energy density spectrum as measured in the surf zone at sensor ADV during March 2000.

La ligne en pointillée indique la limite fréquentielle entre les ondes dites « infragravitaires» et les ondes «hautes fréquences», comprenant les ondes incidentes et les harmoniques. On observe que le spectre s'est profondément modifié en zone de surf, si on le compare au spectre mesuré au niveau du capteur $\mathrm{S} 1$, situé en zone de levée (figure $2 \mathrm{~b}$ ) : le spectre s'est élargi et on ne peut plus considérer que l'énergie est centrée autour d'une fréquence pic à $0.08 \mathrm{~Hz}$, même si celle-ci reste le pic le plus énergétique du spectre. Il est plus judicieux, lorsque le spectre est large d'utiliser la fréquence centroïdale calculée comme étant la moyenne des fréquences pondérées par leur énergie. Dans le cas présent, on trouve une fréquence centroïdale de $0.16 \mathrm{~Hz}(0.10 \mathrm{~Hz}$ dans la figure $2 b)$. Néanmoins, rien ne nous permet à ce stade de déterminer si l'énergie contenue dans les harmoniques est liée à la forme des vagues (les modes sont liés) ou si l'on a réellement formation de nouveaux fronts d'ondes. Or ce point est essentiel pour correctement modéliser la dissipation d'énergic. Des outils d'ordre supérieur, prenant en compte la phase tel que le bispectre doivent alors être envisagés pour compléter les études.

\section{Conclusions}

La zone de surf est une zone particulièrement complexe dans laquelle les caractéristiques de la houle se trouvent fortement modifiées sur de courtes distances (quelques longueurs d'ondes). Or elle est également la zone privilégiée 
pour le transport sédimentaire dont le principal vecteur hydrodynamique est la houle.

De nombreuses formules de transport sont basées sur les caractéristiques de la houle au large, en supposant que celles-ci se conservent le long des lignes de propagation de la houle, or les figures 3 et 4 mettent très clairement en évidence la fragilité de cette hypothèse. En mai 1998, la fusion des fronts d'ondes entraîne une augmentation de la période moyenne d'un facteur 1.2 et d'un facteur 1.5 pour la période significative sur quelques longueurs d'ondes (figure 3) alors qu'en mars 2000, la génération puis la libération d'harmoniques entraînent une diminution de la période moyenne d'un facteur 2 (figure 4). Nul doute que ces modifications engendrent des variations des taux de dissipation au sein de la zone de surf (Sénéchal et al. 2002).

Enfin, le caractère fortement non-linéaire de la houle, à l'approche de la côte, ne permet pas d'interpréter correctement la répartition fréquentielle de l'énergie, compte tenu qu'il ne tient pas compte des relations de phase entre les différents modes.

\section{Références}

[1] BAILARD J.A. (1984)- A simplified method for longshore sediment transport. Proceeding of the $19^{\text {th }}$ Coastal Eng. Conf. pp. 1454-1470.

[2] BEJI S. et BATTJES J.A. (1993). Experimental investigation of wave propagation over a bar. Coastal Eng. 19. p.151-162.

[3] C.E.R.C. (1984)- Shore protection Manual. Vol. I. 1006p.

[4] KAMPHUIS J.W. (1993)- Alongshore Sediment transport rate, closure. Journal of Waterway, Port, Coastal, and Ocean Engineering. 119-3. p. 346-349.

[5] LAFON V., BUTEL R., DUPUIS H., DE MELO APOLUCENO D., MICHEL D., HOWA H. et CASTELLE B.. (2001). Rythmic subtidal and intertidal bar morphology and dynamics in a mixed-energy environment. Part II: physical forcing analysis. En préparation pour J. Coastal Res.

[6] L.C.H.F. (1979)- Action de la houle sur les sédiments. Rapport L.C.H.F. pour le C.N.E.X.O.. 47p.

[7] MASSELINK G. (1998)-Field investigation of wave propagation over a bar and the consequent generation of secondary waves. Coastal Eng. 33. 1-9.

[8] SENECHAL N., BONNETON P. et DUPUIS H. (2001a)-Field observations of irregular wave transformation in the surf zone. Proceeding of Coastal Dynamics-Lund-Suède. p. 62-71.

[9] SENECHAL N., DUPUIS H., BONNETON P., HOWA H. et PEDREROS R. (2001b)- Observation of irregular wave transformation in the surf zone over a gently sloping beach on the French Atlantic coastline. Oceanologica Acta. Vol. 24 (6). p. 545-556.

[10] SENECHAL N., BONNETON P. et DUPUIS H. (2002)-Field experiment on secondary wave generation on a barred beach and the consequent evolution of energy dissipation on the beach face. Coastal Eng. (en révision). 